\title{
Improved free-electron laser stability and performance using a hollow electron beam
}

\author{
Abhishek Pathak and Srinivas Krishnagopal \\ Ion Accelerator Development Division, Bhabha Atomic Research Centre, Mumbai 400085, India
}

(Received 27 November 2017; published 26 July 2018)

\begin{abstract}
We consider the performance of free-electron lasers (FELs) in the oscillator configuration, using a hollow electron beam distribution instead of the usual Gaussian. Using the three-dimensional, timedependent FEL code GENESIS, we show that for FEL oscillators lasing can be achieved over a much broader range of cavity configurations with a hollow electron beam. This occurs because with the hollow electron beam higher-order optical modes and mode competition are suppressed. We also find a substantial increase in the saturated out-coupled power, with the optical mode still remaining the fundamental $\mathrm{TEM}_{00}$ mode. For a hollow electron beam, even with a finite mirror misalignment the transverse optical mode profile remains close to a $\mathrm{TEM}_{00}$ mode but with higher out-coupled power than with perfectly aligned mirrors. Thus, it is preferable to operate FEL oscillators with a hollow electron beam rather than a Gaussian.
\end{abstract}

DOI: 10.1103/PhysRevAccelBeams.21.070702

\section{INTRODUCTION}

Free-electron lasers (FELs) are well known for their transverse coherence and wide range tunability. Among various modes of operation, the oscillator mode is widely preferred at modest current values (10-100 A), for longwavelength FELs [1-18]. Most of these operating FELs use hole out-coupling in order to have efficient out-coupling over a very wide range of wavelengths (an important exception is the JLab FEL). However, introducing a hole in one of the mirrors will perturb the resonator stability. Various theoretical [19-24] and computational [25-27] studies have been performed to investigate FEL stability in terms of both the saturated power and optical mode in the resonator, and are verified with experimental results [28]. Most of these earlier studies explain the stability of the FEL oscillator in terms of the resonator stability parameters $g_{1}, g_{2}$, size of the out-coupling hole, mirror misalignment [26], optical mode competition [24] and so on. It has been proposed that FEL oscillators are better operated near the confocal and concentric configurations. It has also been observed that at shorter wavelengths the stability region is much smaller because of enhanced mode competition [26].

Besides cavity and undulator parameters, beam parameters such as emittance, current, energy and particle distribution contribute significantly to the FEL performance. Studies

Published by the American Physical Society under the terms of the Creative Commons Attribution 4.0 International license. Further distribution of this work must maintain attribution to the author(s) and the published article's title, journal citation, and DOI. carried out by Blank et al. [29] demonstrate that use of a hollow beam leads to higher saturated power in a shorter length for an FEL amplifier, though at low energy. Considering the advantage of reduced space-charge field, hollow beams can be of great assistance in improving the FEL performance in its oscillator configuration as well. Much work has also been done on the design and construction of electron guns that can produce a hollow electron beam $[30,31]$.

In earlier work on FEL resonator stability with a Gaussian electron beam [26] we have shown that the choice of resonator configuration plays a determining role in the lasing process. At shorter wavelengths (10 and $25 \mu \mathrm{m}$ ) the lasing is confined to a small region near the concentric and confocal configurations. In the remaining region the cavity becomes unstable with a higher order Gauss-Laguerre TEM mode. Issues with both mode beating and higher order optical mode limit the parameter space, especially the wavelength and $g$ parameter, over which lasing can be obtained. The introduction of cavity mirror misalignment perturbs the lasing action in an interesting fashion: the out-coupled power is higher for a finite mirror tilt, instead of no tilt, but at the cost of a higher order Gauss-Laguerre optical mode.

Here we perform fully three-dimensional, time dependent, simulations using the codes GENESIS [32] and OPC [33], to analyze the effect of a hollow electron beam on FEL performance. We consider the oscillator configuration, Fig. 1, for the FEL and study the issues related to saturated power, mode competition, transverse optical mode and region of stability using a hollow electron beam. We also look at the consequences of mirror misalignment in the cavity. We show that use of a hollow beam improves the resonator stability and performance. 


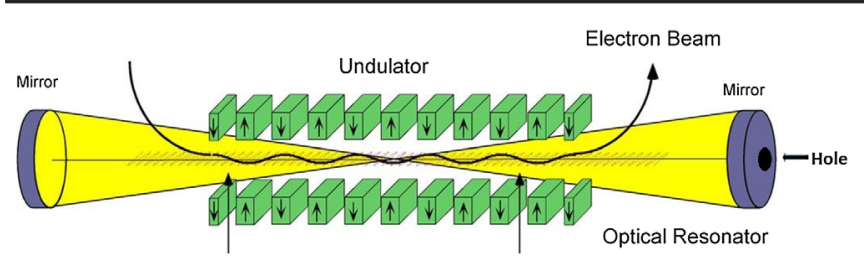

FIG. 1. Schematic showing the oscillator configuration of the free-electron laser. Radiation is out-coupled through a hole in the downstream mirror.

In the next section we summarize the FEL parameters used in these simulations. In Sec. III we present simulation results for an FEL oscillator, using both hollow and Gaussian electron beams. We look at the dependence on the cavity configuration (by varying the cavity length), and investigate in detail how mode competition is suppressed with a hollow electron beam. We also look at the effect of mirror tilt. Finally, in Sec. IV we present a discussion on FELs that do not use hole out-coupling (such as the JLab FEL and proposed x-ray FEL oscillators), before concluding.

\section{FEL PARAMETERS}

We considered a long wavelength FEL oscillator with an out-coupling hole in the downstream mirror, and with a $2 \mathrm{~m}$ long planar undulator with flat pole faces having a period of $50 \mathrm{~mm}$. The length of the cavity was varied between 6.15 to $12.3 \mathrm{~m}$ in order to cover the entire range of cavity configurations, from confocal to concentric. The undulator was placed at the center of the cavity, and power was outcoupled through a hole in the downstream mirror. Other parameters of interest are listed in Table I. We considered initial electron beam distributions that were Gaussian as well as hollow, as shown in Fig. 2.

The code OPC uses a Perl script where for every roundtrip it calls GENESIS (in a FOR loop) and finally dumps the data at the end of a specified number of passes. For the beam matching we considered a circular electron beam with upright ellipse in $\mathrm{x}-\mathrm{x}$ ' and $\mathrm{y}-\mathrm{y}$ ' phase space $(\alpha=0)$, at the entrance to the planar undulator. We varied the electron rms transverse sizes $(=\sqrt{\beta \epsilon})$ at the entrance of the undulator in the GENESIS input file, thus varying the $\beta$-function, and observed the saturated power after a specified number of passes (100). We chose that value of the input beam size for which this saturated power was maximum, indicative of the best practical matching. This value of the rms size was $0.6 \mathrm{~mm}$, leading to a total beam size, for the Gaussian beam, of $2.23 \mathrm{~mm}$ (given in Table I). For the hollow beam, this value was taken as the outer radius of the hollow beam. The inner radius was taken as $0.8 \mathrm{~mm}$, after optimizing it with respect to the saturated power (for the same outer radius).

Figure 3 shows the variation of the transverse size of the electron beam along the undulator for $\lambda=10 \mu \mathrm{m}$ for both Gaussian and hollow beams. It can be seen that the vertical
TABLE I. FEL parameters for the simulations.

\begin{tabular}{lc}
\hline \hline Parameter & Value \\
\hline Undulator parameter $\left(K=\frac{e B \lambda_{u}}{2 \pi m c}\right)$ & 0.637 \\
Undulator type & Linear \\
Period $\left(\lambda_{u}\right)(\mathrm{mm})$ & 50 \\
Number of periods & 40 \\
$\gamma$ & Tuned for wavelengths \\
Relative energy spread & $0.3 \%$ \\
Gaussian beam matched & 2.23 \\
$\quad$ beam size (mm) & \\
Hollow beam outer radius (mm) & 2.23 \\
Hollow beam inner radius (mm) & 0.80 \\
X emittance (mm-mrad) & 20 \\
Y emittance (mm-mrad) & 20 \\
Beam current (A) & 100 \\
Beam longitudinal distribution & Gaussian $(6 \sigma)$ \\
Beam rms length (mm) & 3 \\
Radiation wavelength $(\mu \mathrm{m})$ & $10,25,50$ \\
Seed power (MW) & 1 \\
Seed radiation transverse profile & Gaussian \\
Radius of curvature of mirror (m) & 6.15 \\
Mirror cross-section radius (mm) & 23 \\
Out-coupling hole radius (mm) & 4 \\
Out-coupling mirror tilt (mrad) & $0-5$ \\
Number of grid points along x and y & 512 \\
Grid spacing along x and y (mm) & 0.4 \\
Number of longitudinal slices & 250 and 120 \\
Number of macroparticles per slice & 102,400 \\
\hline \hline
\end{tabular}

size has a waist at the center of the undulator, as may be expected from the undulator focusing. The horizontal size increases slightly, by around $0.38 \mathrm{~mm}$, through the undulator for both hollow and Gaussian beam, because there is no undulator focusing in that direction. We could have attempted further optimization by adjusting the value of $\alpha_{x}$, but since that is not essential to the main thesis of this paper, that hollow electron beams lead to better FEL performance compared to Gaussian, we have not pursued that optimization at this stage.

The simulation parameters given in Table I were chosen so that the results are not artifacts of numerical noise or error.



FIG. 2. Input electron distribution to FEL oscillator: (a) Gaussian; (b) hollow. 


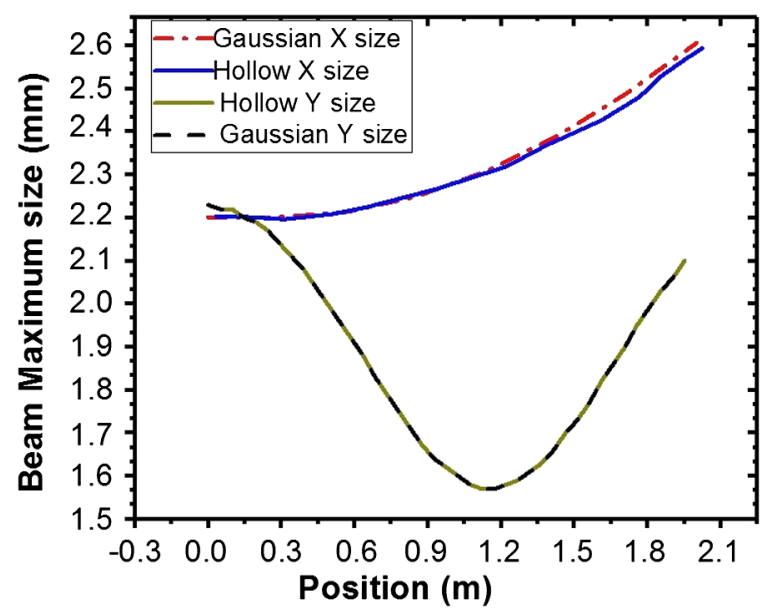

FIG. 3. Variation of the transverse size of the electron beam along the undulator for $\lambda=10 \mu \mathrm{m}$ for Gaussian and hollow beams.

\section{FEL INTERACTION WITH A HOLLOW BEAM}

\section{A. Cavity length variation}

We performed simulations with Gaussian as well as hollow, specifically Gauss-Laguerre $\mathrm{LG}_{01}$, initial electron beam distributions. We first investigated the dependence of saturated power and equilibrium optical mode on the cavity stability parameter $g=1-(l / R)$, where $l$ is the cavity length and $\mathrm{R}$ is the radius of curvature of the mirror. We considered a symmetric resonator and varied the cavity length from 6.15 to $12.3 \mathrm{~m}$ (from the confocal to the concentric configuration), while keeping the radius of curvature of the mirror fixed $(6.15 \mathrm{~m})$. As FELs are known for their wide bandwidth, we performed this study for 10 , 25 and $50 \mu \mathrm{m}$ laser wavelengths.

We looked at the saturated out-coupled power as a function of the $g$-parameter (or equivalently the resonator length), for the Gaussian as well as hollow beam. The results are shown in Fig. 4. With a Gaussian electron beam the cavity is stable only near the confocal and concentric configurations. However, with the hollow beam lasing occurs over a wide range of resonator lengths. For a wavelength of $10 \mu \mathrm{m}$, Fig. 4(a) shows that lasing with a hollow electron beam occurs throughout the entire region from $g=-1$ to $g=0$, i.e. from the confocal to the concentric configuration. However, with the Gaussian electron beam lasing occurs only near the concentric and confocal extremes, and at one pocket, between $g=-0.8$ and $g=-0.65$. Over most of the range (around 65\%) there is simply no lasing with a Gaussian electron beam. Moreover, the hollow electron beam performs better in terms of the intensity of out-coupled radiation. The outcoupled power never drops below $15 \mathrm{MW}$ over the entire configuration range, reaching as high as $40 \mathrm{MW}$. In contrast, with a Gaussian electron beam, the power varies between 7.5 and $30 \mathrm{MW}$. At almost every resonator

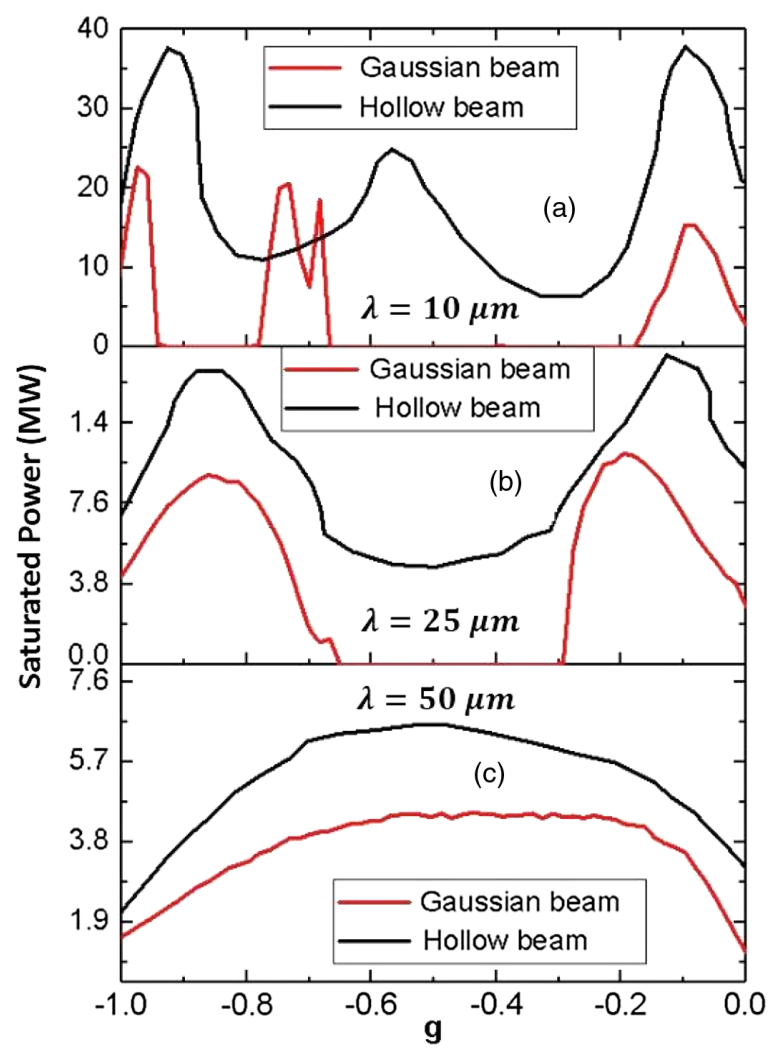

FIG. 4. Variation of out-coupled saturated power with cavity stability parameter $g$, for Gaussian and hollow electron beams, for (a) $\lambda=10 \mu \mathrm{m}$, (b) $\lambda=25 \mu \mathrm{m}$, and (c) $\lambda=50 \mu \mathrm{m}$.

configuration, the hollow electron beam generates more out-coupled laser power than the Gaussian electron beam.

Figure 4(b) shows similar behavior at a wavelength of $25 \mu \mathrm{m}$. Again, with a hollow electron beam there is lasing over the entire configuration range, from confocal to concentric, whereas with the Gaussian electron beam lasing is confined to near the confocal and concentric configurations; there is no lasing between $g=-0.65$ and $g=-0.3$. In addition, at every resonator configuration, the hollow electron beam generates more out-coupled laser power than the Gaussian electron beam. The hollow electron beam gives a maximum out-coupled power of $16 \mathrm{MW}$, whereas with the Gaussian electron beam this number is only $9 \mathrm{MW}$.

Thus, at shorter and intermediate wavelengths the hollow electron beam clearly provides better performance than the Gaussian beam, with lasing over the full range of resonator configurations, and with higher out-coupled power.

At the longer wavelength of $50 \mu \mathrm{m}$, Fig. 4(c), the situation is slightly different. Both the hollow as well as Gaussian electron beams provide lasing over the entire configuration space, from confocal to concentric. However, even in this case the hollow electron beam performs better in that it provides much greater out-coupled laser power; up to a maximum of $8 \mathrm{MW}$, compared to only $4 \mathrm{MW}$ for the Gaussian beam. 

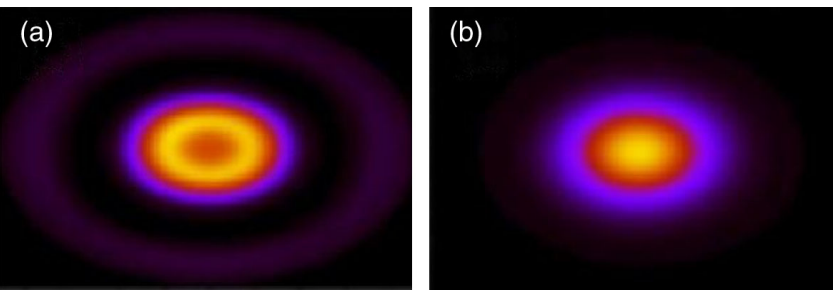

FIG. 5. Transverse optical field profile at the out-coupled mirror: for the (a) Gaussian, and (b) hollow, electron beams, both at $g=-0.6$ and $\lambda=10 \mu \mathrm{m}$.

The reason for the better performance of the hollow beam can be understood by looking at the optical mode of the FEL. For example, consider a wavelength of $10 \mu \mathrm{m}$ and $g=-0.6$, Fig. 4(a), where the hollow electron beam produces lasing but the Gaussian does not. The optical mode at the downstream mirror, for both cases, is shown in Fig. 5. For the Gaussian beam, Fig. 5(a), the mode is a higher order Gauss-Laguerre mode which has lesser intensity at the center, from where the power is out-coupled. However, the moment we use a hollow beam, Fig. 5(b), the mode improves to a Gaussian-like mode, with a maximum at the center and intensity decreasing with radius.

These results show that use of a hollow electron beam leads to a significant improvement in the FEL performance in terms of the out-coupled power as well as the transverse optical mode that is now available throughout, between the confocal and concentric configurations. This makes the FEL facility more useful to users, since high power radiation can now be provided over the entire range of cavity configurations and at all wavelengths.

\section{B. Suppression of mode competition}

Degenerate eigenmodes can cause mode competition in the resonator, which is known to affect lasing. To circumvent this problem, to a limited extent, techniques like active and passive control are used.

As shown above, hollow beams produce excellent results in terms of saturated power for a given cavity length. However, we know that higher out-coupled power has a direct relation with the equilibrium mode in the cavity. In order to better understand the saturation process in terms of the stability of the eigenmode as well as saturated outcoupled power, we analyzed the behavior of these optical modes in terms of their power and mode stability, for $\lambda=10 \mu \mathrm{m}, \lambda=25 \mu \mathrm{m}$ and $\lambda=50 \mu \mathrm{m}$, as a function of cavity round-trip. The results of these simulations are shown in Fig. 6; note that for each plot the power is normalized to its maximum value, so that each plot varies from 0 to 1 , and quantitative comparisons cannot be made between the plots.

At a wavelength of $10 \mu \mathrm{m}$, Fig. 6(a) shows that with a Gaussian electron beam the optical power oscillates with each round-trip, which is a signal of mode beating, and

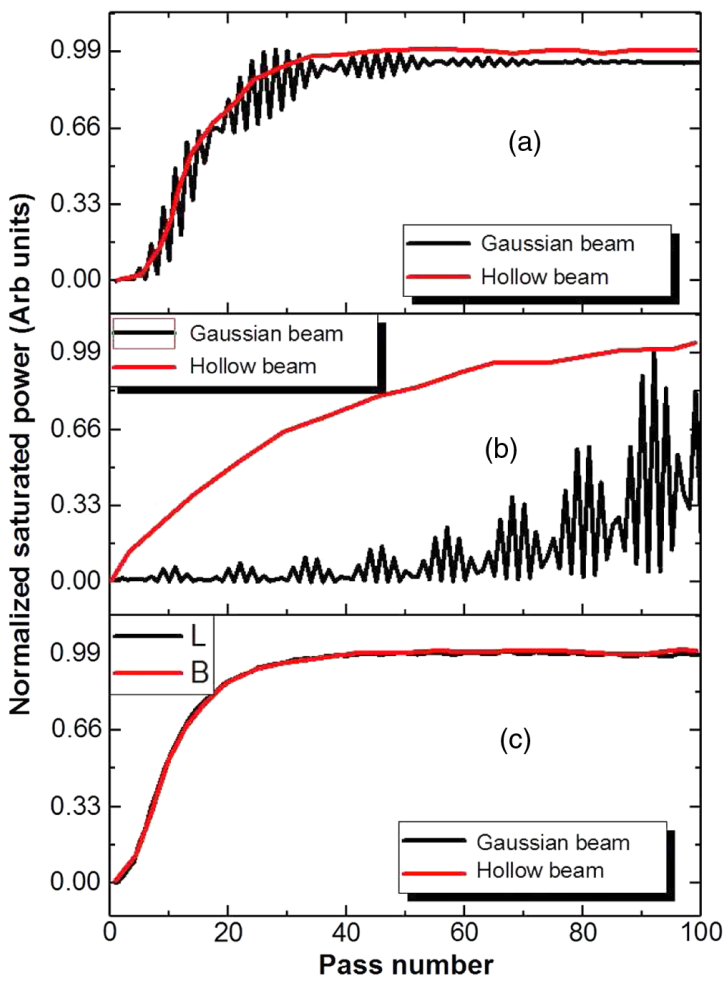

FIG. 6. Out-coupled power as a function of pass number in the FEL resonator for the hollow and Gaussian beams, for (a) $\lambda=10 \mu \mathrm{m}$, (b) $\lambda=25 \mu \mathrm{m}$ and (c) $\lambda=50 \mu \mathrm{m}$. In all cases $g=-0.6$.

saturation is reached after around 80 passes, when mode beating has disappeared. For the hollow beam, on the other hand, the out-coupled power increases without any oscillation with successive round-trips, leading to a saturation just after 45 passes. At a wavelength of $25 \mu \mathrm{m}$, Fig. 6(b), with a Gaussian electron beam we again observe beating of the optical mode, this time without achieving saturation. The situation dramatically improves with the use of a hollow electron beam with no oscillation and saturation in out-coupled power. Finally, at a longer wavelength of $50 \mu \mathrm{m}$, the increase and saturation of the out-coupled power is almost identical for the Gaussian and hollow electron beams. In particular, no mode beating is seen with the Gaussian electron beam, and in both cases power saturates at around the same value and after the same number of round-trips.

A clearer understanding of mode beating emerges from looking at the optical mode profiles on the downstream mirror. At a wavelength of $10 \mu \mathrm{m}$, corresponding to Fig. 6(a), we looked at the mode profiles on three successive passes $(60,61$ and 62), for both beams. These are shown in Figs. 7(a)-7(c) for the Gaussian electron beam and in Figs. 7(d)-7(f) for the hollow electron beam. It can be seen that for the Gaussian electron beam the optical mode oscillates between different higher-order modes. This mode oscillation is reflected in the power 

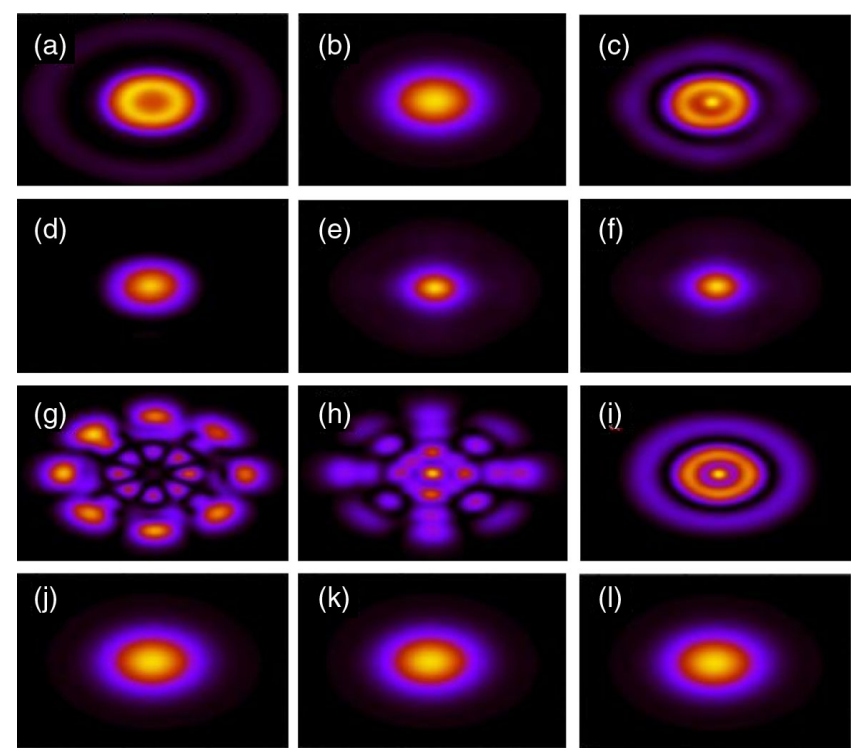

FIG. 7. Transverse optical mode as a function of pass number for Gaussian and hollow electron beams for three consecutive passes (60, 61 and 62). (a), (b), (c) and (g), (h), (i) are for the Gaussian electron beam and at wavelengths of 10 and $25 \mu \mathrm{m}$ respectively, while (d), (e), (f) and (j), (k), (l) are for the hollow electron beam and at wavelengths of 10 and $25 \mu \mathrm{m}$ respectively. In all cases $g=-0.6$. The scale for all the plots are from -23 to $23 \mathrm{~mm}$ in both $\mathrm{x}$ and $\mathrm{y}$ directions.

oscillation in Fig. 6(a). For the hollow beam, the mode is always Gaussian, and therefore the power in Fig. 6(a) evolves smoothly.

Similarly, at a wavelength of $25 \mu \mathrm{m}$, corresponding to Fig. 6(b), the mode profiles on three successive passes (60, 61 and 62), are shown in Figs. 7(g)-7(i) for the Gaussian beam, and Figs. 7(j)-7(l) for the hollow beam. Again, for the Gaussian beam the modes on all three passes are different higher-order modes, in this case quite dramatically so. As a consequence there are large oscillations in the out-coupled power in Fig. 6(b). However, for the hollow electron beam the mode is always Gaussian, and therefore the power evolves smoothly.

In order to have a quantitative comparison of the power, Fig. 8 shows the power variation of Fig. 6(a), but now without normalization. Both, the intracavity power and the out-coupled power are shown for comparison. It can be seen that the hollow beam produces much higher power than the Gaussian - whether in terms of the intracavity power or the out-coupled power. For the hollow beam, the saturated intracavity power is around $46 \mathrm{MW}$, while the out-coupled power is around $21 \mathrm{MW}$. For the Gaussian beam, these numbers are only 4.55 and $0.4 \mathrm{MW}$, respectively.

Figure 8 also shows the modes on the downstream outcoupling mirror at passes 30,60 and 80. For the hollow beam it can be seen that the mode is always the same, whereas for the Gaussian beam the mode is different at each pass. For both beams these modes are consistent with those

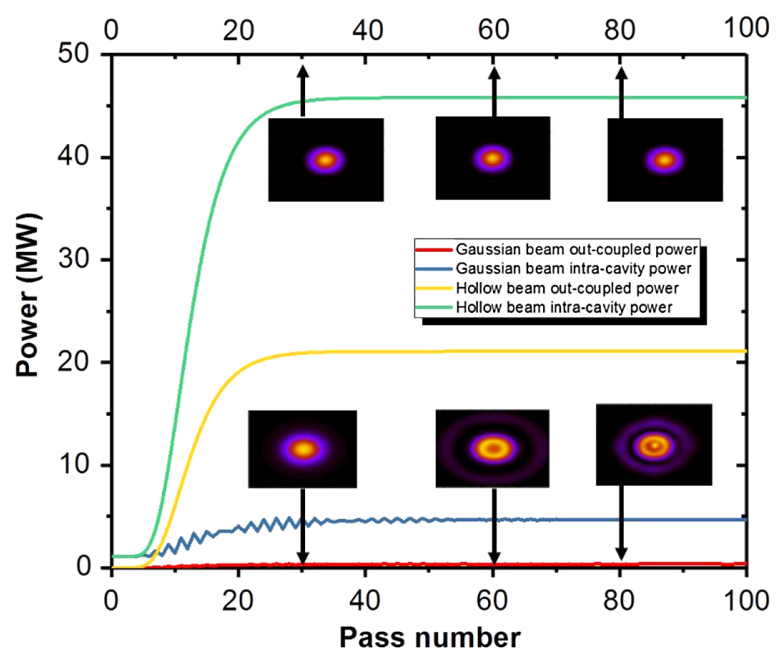

FIG. 8. Out-coupled and intracavity power as a function of pass number for the Gaussian and hollow beams. Also shown are the transverse modes at the out-coupling mirror after the 30th, 60th and 80th passes, for both beams. With $g=-0.6$ and $\lambda=10 \mu \mathrm{m}$.

shown in Fig. 7, which were for three consecutive passes, 60,61 and 62 .

It is instructive to look at the out-coupling fraction, which is the ratio of the out-coupled power to the intracavity power, for the two beams. From Fig. 8 it can be seen that for the hollow beam the out-coupling fraction is $45 \%$, while for the Gaussian it is less than $20 \%$. This difference is clearly because of the difference in the mode structure.

From Fig. 8 it can also be seen that the small-signal gain is greater for the hollow beam compared to the Gaussian. This is because for the latter, the higher-order optical mode has a poorer overlap with the Gaussian electron beam, compared to the overlap of the higher-order electron beam with the Gaussian radiation beam. This in turn is because for the hollow beam the hole at the center is very small, around $0.5 \mathrm{~mm}$ in radius, compared to the size of the radiation mode (radius of around $10 \mathrm{~mm}$ ), so that the overlap is still pretty significant.

From these results it is clear that hollow electron beams are especially effective at shorter wavelengths at controlling mode competition and beating, and delivering a nearGaussian optical mode. As a consequence, lasing occurs at all wavelengths, and the out-coupled power increases and saturates smoothly, in few tens of passes.

\section{Effect of mirror tilt}

Most stability analyses performed on FEL resonators consider a symmetric cavity with the assumption that the upstream and downstream mirrors are parallel with respect to each other. In practice these components are assembled physically and the possibility of misalignment cannot be ignored. Earlier studies with a Gaussian electron beam [26] have shown that an FEL in the confocal configuration is more affected by mirror tilt than in the concentric 


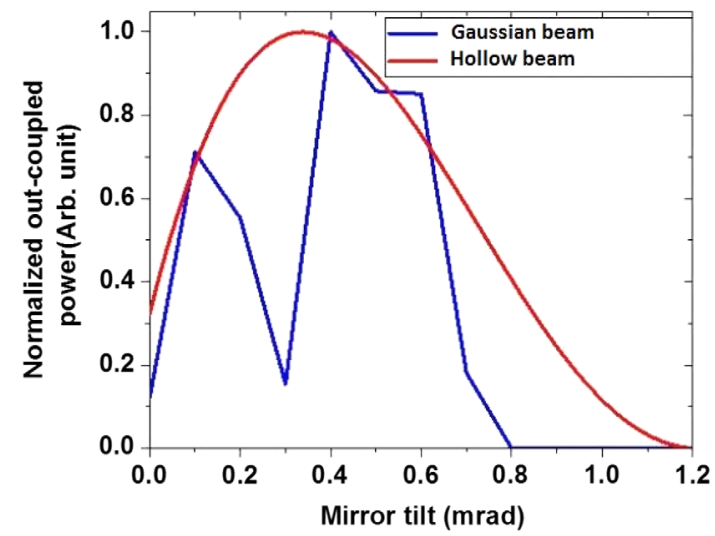

FIG. 9. Out-coupled power as a function of mirror tilt with Gaussian and hollow electron beams at $\lambda=10 \mu \mathrm{m}$, for a confocal resonator.

configuration. It was also found that for all wavelengths, the maximum out-coupled power obtained in the confocal configuration is for a nonzero mirror tilt, but this is at the cost of a higher-order optical mode.

Here we repeat the same studies with a hollow electron beam instead of a Gaussian beam. At a wavelength of $10 \mu \mathrm{m}$, for a confocal resonator, Fig. 9 shows the normalized out-coupled power as a function of mirror tilt, where we have assumed equal tilts in both transverse directions. Note that for each curve the power is normalized to its maximum value, so that each curve varies from 0 to 1 , and quantitative comparisons cannot be made between the plots.

One can see from Fig. 9 that with a Gaussian electron beam the out-coupled power performs an oscillation with increasing mirror tilt and is maximum at a tilt of $0.42 \mathrm{mrad}$; the absolute value of the power is $8.3 \mathrm{MW}$. As the mirror tilt is changed, there are sharp changes in the out-coupled power; therefore, even a slight misalignment of the mirrors can lead to a large change in the out-coupler power. At large values of mirror tilt, over $0.8 \mathrm{mrad}$, lasing is extinguished.

With a hollow electron beam the out-coupled power increases gradually with increase in the mirror tilt, with a maximum value at $0.36 \mathrm{mrad}$ (where the absolute value of the power is $79 \mathrm{MW}$ ), and again drops to zero at $1.2 \mathrm{mrad}$. There are no abrupt variations in the out-coupled power. A direct comparison of the two curves in Fig. 9 clearly suggests that operating an FEL with a hollow electron beam provides more tolerance towards mirror misalignment for resonators operating in the confocal configuration. Also, the outcoupled power is much higher with the hollow beam.

Analyzing the optical field from the point of view of quality of the transverse optical mode, we can see from Figs. 10(a) and 10(c) that without mirror tilt the optical beam is essentially Gaussian for both hollow as well as Gaussian electron beams. However, with nonzero tilt the transverse optical modes that we obtain in the two cases are poles apart, as shown in Figs. 10(b) and 10(d). The mode obtained with a Gaussian beam is closer to Gauss-Laguerre
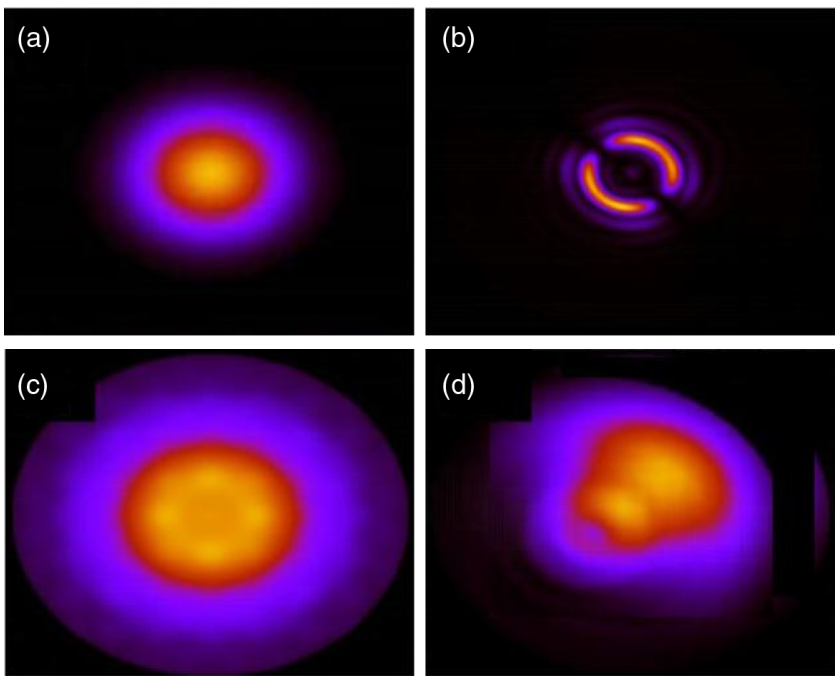

FIG. 10. Transverse optical field profile at the out-coupled mirror: (a) for the Gaussian electron beam with zero tilt, (b) for the Gaussian electron beam with a tilt of $0.42 \mathrm{mrad}$, (c) for a hollow electron beam with zero tilt and (d) for a hollow electron beam with the tilt of $0.42 \mathrm{mrad}$, all at $g=-0.6$ and $\lambda=10 \mu \mathrm{m}$.

$G L_{03}+G L_{01}$ mode, while the one obtained with a hollow electron beam is closer to the fundamental Gauss-Laguerre $G L_{00}$ (Gaussian) mode. These results show that though for both the Gaussian hollow electron beams the out-coupled power is maximum (and almost equal) for a finite tilt of around $0.4 \mathrm{mrad}$, the transverse optical mode obtained in the case of hollow beam is more promising in terms of practical application as it is closer to the fundamental $\mathrm{TEM}_{00}$ mode.

\section{DISCUSSION AND CONCLUSION}

It should be noted that we have restricted our study to hole out-coupled FEL oscillators, because almost all operating FELs use hole out-coupling; a choice driven by the need to have substantial out-coupled power over a wide range of operating wavelengths. It is the introduction of the hole in one of the mirrors that leads to the optical cavity supporting higher-order modes. Therefore the issue of higher-order modes and mode competition is endemic to operating FELs. Our work suggests that using hollow beams could solve this problem without sacrificing the advantages of hole out-coupling.

However, experiments have been performed at Jefferson Laboratory, at a wavelength of $1.6 \mu \mathrm{m}$, using transmissive out-coupling [34], where the out-coupled mode is essentially Gaussian. This experiment has been modeled using MEDUSA and OPC, and the simulations seem to agree with the experiments $[35,36]$.

Studies on x-ray FEL oscillators [37-39] use more complicated resonator geometries, in which there is no hole out-coupling. Here too the modes are essentially Gaussian. 
In summary, we have performed three-dimensional, time-dependent simulations using GENESIS and OPC to investigate the advantages of using a hollow electron beam instead of a Gaussian in the FEL oscillator. A hollow electron beam results in an equilibrium optical mode that is close to Gaussian. As a result, lasing can be found at all resonator configurations and at all wavelengths; with a Gaussian beam it is invariably the formation of a higherorder optical mode that terminates lasing and limits performance. For operating FEL facilities this is an important advantage, allowing lasing over the complete range of wavelengths that the FEL can be tuned to. In addition, the hollow electron beam provides higher power at almost all wavelengths. Our study of FEL oscillators to understand their tolerance towards cavity mirror misalignments showed that we obtain the maximum saturated outcoupled power for a nonzero tilt value, as we obtain with a Gaussian electron beam. However, with a hollow beam, instead of a higher order Gauss-Laguerre mode that we obtain with a Gaussian beam, we obtain an optical distribution that is close to the $\mathrm{TEM}_{00}$ mode. Overall, therefore, we find that there are substantial advantages to employing hollow electron beams in FELs-enough to warrant experimental studies.

[1] P. Neyman, W. B. Colson, S. C. Gottshalk, A. M. M. Todd, J. Blau, and K. Cohn, Free electron lasers, in the 38th International Free Electron Laser Conference (FEL'17), Santa Fe, NM, 2017 (JACOW, Geneva, Switzerland, 2018), pp. 204-209.

[2] P. Guimbal et al., First results in the saturation regime with the ELSA FEL, Nucl. Instrum. Methods Phys. Res., Sect. A 341, 43 (1994).

[3] P. Guimbal et al., High efficiency results at $23 \mathrm{~m}$ on the ELSA FEL and operation with the MONA wiggler, Nucl. Instrum. Methods Phys. Res., Sect. A 358, 240 (1995).

[4] J. G. Karssenberg, P. J. van der Slot, J. W. Verschuur, I. V. Volokhine, and K. J. Boller, FEL-oscillator simulations with GENESIS 1.3, in 28th FEL Conference, Berlin, Germany (JACOW, Geneva, Switzerland, 2006), pp. 407-410.

[5] W. Schllkopf, S. Gewinner, H. Junkes, A. Paarmann, G. von Helden, H. P. Bluem, and A. M. Todd, The new IR and THz FEL facility at the Fritz Haber Institute in Berlin, Proc. SPIE Int. Soc. Opt. Eng. 9512, 95121L (2015).

[6] H. Zen et al., Present status of Kyoto University free electron laser, in Proceedings of the 35th International FEL Conference (JACOW, Geneva, Switzerland, 2013), pp. 711-714.

[7] K. Hayakawa et al., Operation of near-infrared FEL at Nihon University, in Proceedings of the FEL 27th International LINAC Conference, Lubeck, Germany (JACOW, Geneva, Switzerland, 2004), pp. 90-92.

[8] N. A. Vinokurov et al., Novosibirsk free electron laser facility: Two-orbit ERL with two FELs, in Proceedings of
FEL-2009,Liverpool, UK, 2009, TUOD01. 2010 (JACOW, Geneva, Switzerland), pp. 447-451.

[9] G. N. Kulipanov et al., Novosibirsk free electron laser facility description and recent experiments, IEEE Trans. Terahertz Sci. Technol. 5, 798 (2015).

[10] H. Horiike, N. Tsubouchi, K. Awazu, M. Asakawa, and M. Heya, Status of the Institute of Free-Electron Laser, Osaka University, Jpn. J. Appl. Phys. 41, 10 (2002).

[11] R. Kato et al., High power terahertz FEL at ISIR, Osaka University, in Proceedings of the International Particle Accelerator Conference, Kyoto, Japan (ICR, Kyoto, 2010), Vol. 10, p. 2209.

[12] G. Ramian, The new UCSB free-electron lasers, Nucl. Instrum. Methods Phys. Res., Sect. A 318, 225 (1992).

[13] E. Grosse, U. Lehnert, P. Michel, R. Schlenk, W. Seidel, A. Wolf, U. Willkommen, U. D. Wohlfarth, and R. Wuensch, A far-infrared FEL for the radiation source ELBE, in Proceedings of the 27th Free Electron Laser Conference, Stanford, California, 2005 (JACOW, Geneva, Switzerland, 2005), pp. 21-26.

[14] N. R. Thompson, D. J. Dunning, J. A. Clarke, M. Surman, A. D. Smith, Y. Saveliev, and S. Leonard, First lasing of the ALICE infrared free-electron laser, Nucl. Instrum. Methods Phys. Res., Sect. A 680, 117 (2012).

[15] M. Brunken, S. Döbert, R. Eichhorn, H. Genz, H.-D. Gräf, H. Loos, A. Richter, B. Schweizer, A. Stascheck, and T. Wesp, First lasing of the Darmstadt cw free electron laser, Nucl. Instrum. Methods Phys. Res., Sect. A 429, 21 (1999).

[16] T. Takii et al., First lasings at IR and FIR range using the FELI FEL facility 4, in Proceedings of the Free Electron Laser Conference (JACOW, Geneva, Switzerland, 1998), pp. 21-25.

[17] R. Hajima, T. Shizuma, M. Sawamura, R. Nagai, N. Nishimori, N. Kikuzawa, and E. J. Minehara, First demonstration of energy-recovery operation in the JAERI superconducting linac for a high-power free-electron laser, Nucl. Instrum. Methods Phys. Res., Sect. A 507, 115 (2003).

[18] Y. U. Jeong et al., First lasing of the KAERI compact farinfrared free-electron laser driven by a magnetron-based microtron, Nucl. Instrum. Methods Phys. Res., Sect. A 475, 47 (2001).

[19] A. Curcio, G. Dattoli, E. Di Palma, and A. Petralia, Free electron laser oscillator efficiency, Opt. Commun. 425, 29 (2018).

[20] K. Li and H. Deng, Gain cascading scheme of a freeelectron-laser oscillator, Phys. Rev. Accel. Beams 20, 110703 (2017).

[21] Q. K. Jia, Parameter design considerations for an oscillator IR-FEL, Chin. Phys. C 41, 018101 (2017).

[22] M. Xie and K.-J. Kim, Hole coupling resonator for free electron lasers, Nucl. Instrum. Methods Phys. Res., Sect. A 304, 792 (1991).

[23] M. Xie and K.-J. Kim, Performance of hole-coupling resonator in the presence of asymmetric modes and FEL gain, Nucl. Instrum. Methods Phys. Res., Sect. A 318, 877 (1992).

[24] S. Krishnagopal and A. M. Sessler, Stability of resonator configurations in the presence of free-electron laser interactions, Opt. Commun. 98, 274 (1993). 
[25] S. Krishnagopal, M. Xie, and K.-J. Kim, Suppression of mode beating in a saturated hole-coupled FEL oscillator, Nucl. Instrum. Methods Phys. Res., Sect. A 331, 654 (1993).

[26] A. Pathak and S. Krishnagopal, Resonator stability and higher-order modes in free-electron laser, Phys. Rev. ST Accel. Beams 17, 080703 (2014).

[27] S. A. Samant and S. Krishnagopal, Stability analysis of free-electron laser resonators, in Proceedings of the International Particle Accelerator Conference, Kyoto, Japan (ICR, Kyoto, 2010), p. 2170.

[28] R. Prazeres, J. M. Berset, R. Chaput, F. Glotin, D. A. Jaroszynski, and J. M. Ortega, CLIO, an infrared free electron laser facility, Nucl. Instrum. Methods Phys. Res., Sect. B 89, 54 (1994).

[29] M. Blank, H. P. Freund, R. H. Jackson, D. E. Pershing, and J. M. Taccetti, Simulation of an annular beam free-electron laser, Nucl. Instrum. Methods Phys. Res., Sect. A 375, 169 (1996).

[30] M. Friedman and M. Ury, Production and focusing of a high power relativistic annular electron beam, Rev. Sci. Instrum. 41, 1334 (1970).

[31] G. Stancari et al., Development of hollow electron beams for proton and ion collimation, in Proceedings of the International Particle Accelerator Conference, Kyoto, Japan (ICR, Kyoto, 2010), p. 1698.

[32] S. Reiche, GENESIS 1.3: A fully 3D time-dependent FEL simulation code, Nucl. Instrum. Methods Phys. Res., Sect. A 429, 243 (1999).
[33] OPC design team, http://lpno.tnw.utwente.nl/index.php? $\bmod =$ research and projectid $=21$ andcontentid $=11$.

[34] G. R. Neil, C. Behre, S. V. Benson, M. Bevins, G. Biallas, J. Boyce, and R. Evans, The JLab high power ERL light source, Nucl. Instrum. Methods Phys. Res., Sect. A 557, 9 (2006).

[35] H. P. Freund, P. J. van der Slot, D. L. A. G. Grimminck, I. D. Setija, and P. Falgari, Three-dimensional, timedependent simulation of free-electron lasers with planar, helical, and elliptical undulators, New J. Phys. 19, 023020 (2017).

[36] P. J. van der Slot, H. P. Freund, W. H. Miner Jr., S. V. Benson, M. Shinn, and K. J. Boller, Time-Dependent, Three-Dimensional Simulation of Free-Electron-Laser Oscillators, Phys. Rev. Lett. 102, 244802 (2009).

[37] W. Qin, K. Bane, Y. Ding, S. Huang, Z. Huang, K. J. Kim, and T. Maxwell, Start-to-end simulations for an X-ray FEL oscillator at the LCLS-II and LCLS-II-HE, in Proceedings of the 38th International Free Electron Laser Conference (FEL'17), Santa Fe, NM (JACOW, Geneva, Switzerland, 2017), pp. 247-250.

[38] K. J. Kim, Y. Shvydko, and S. Reiche, A Proposal for an $\mathrm{X}$-Ray free-Electron Laser Oscillator with an EnergyRecovery Linac, Phys. Rev. Lett. 100, 244802 (2008).

[39] J. Dai, H. Deng, and Z. Dai, Proposal for an X-Ray Free Electron Laser Oscillator with Intermediate Energy Electron Beam, Phys. Rev. Lett. 108, 034802 (2012). 